\title{
Multi-Element Aperiodic Array Synthesis by Compressive Sensing
}

\author{
C. Bencivenni, M. V. Ivashina, and R. Maaskant
}

\begin{abstract}
In recent years, Compressive Sensing has attracted considerable attention in various areas of antennas and electromagnetics, including the synthesis of sparse array antennas. The CS synthesis of arrays achieves higher accuracy than analytical methods and allows for the fast and deterministic design of large complex arrays, without resorting to computationally expensive Global Optimization methods. The CS approach presented here has been previously studied by the authors for the design of maximally sparse arrays in the presence of mutual coupling effects, beam scanning degradation, as well as the imposition of symmetries for design modularity. In this manuscript the authors demonstrate another (yet unexplored) capability of such an approach, i.e., to incorporate different element types and determine their optimum combination in the course of the array synthesis procedure. Numerical examples are illustrated for large arrays comprising uniform circular aperture elements and operating in a SATCOM multi-beam scenario. It is shown that by exploiting this capability it is possible to simultaneously reduce the number of elements and gain scan loss.
\end{abstract}

Index Terms-sparse array, Compressive Sensing, wide angle scanning, multi element type, uniform circular aperture

\section{INTRODUCTION}

Antenna arrays offer attractive capabilities over singleelement antennas, though are typically expensive due to the large number of elements and electronic components involved. Aperiodic array antennas can substantially reduce the number of elements and associated costs with respect to regular arrays but their design is challenging [1]. Several synthesis methods have been proposed, yet aperiodic array design techniques are not as mature as those in use for their regular array counterparts. These methods are often either: (i) accurate but computationally expensive (e.g. Genetic Optimization techniques), or; (ii) efficient but simplified (e.g. analytical techniques). Examples of Genetic Optimization techniques include Genetic Algorithms [2], Particle Swarms [3], Ant Colonies [4] and Invasive Weed Optimizations [5], while some analytical techniques worth mentioning are the Matrix Pencil Method [6], Almost Different Sets [7], the Auxiliary Array Factor [8] Poisson Sum Formula [9], the Iterative Fourier Technique [10], and Density Tapered method [11].

Compressive Sensing (CS) has been recently applied to the synthesis of sparse antenna arrays. The method can optimize large maximally sparse antenna array problems in a fast, deterministic and flexible way [12]. In previous publications ( [13], [14]) the authors have: (i) extended the original formulation in [12] to the multi-beam scenario; (ii) combined it with

C. Bencivenni, and M. V. Ivashina and R. Maaskant are with the Department of Signals and Systems at Chalmers University of Technology, Göteborg, Sweden, e-mail: carlo.bencivenni@chalmers.se, marianna.ivashina@chalmers.se, rob.maaskant@chalmers.se. acceleration techniques exploiting the array layout symmetry and enabling its modular design; and (iii) hybridized the original iterative optimization procedure with a full-wave EM analysis, so as to include the effects of the antenna element geometry and mutual coupling into the design process. This extended approach has been studied for arrays of strongly coupled antennas elements, such as dipoles, as well as large planar arrays of pipe horns [13], [14].

In this manuscript, we propose a further extension to our optimization framework, namely the synthesis of multielement arrays. The idea has been already investigated by other authors, within the realm of different approaches [15], albeit in a deterministic manner and mostly in connection with isophoric arrays. The deployment of multiple types of antenna elements introduces additional degrees of freedom in the optimization problem and may therefore result in a further reduction in the number of elements owing to the larger element sizes. The method will be demonstrated for the synthesis of large planar arrays for SATCOM applications. The considered element types are Uniform Circular Apertures with variable aperture size.

\section{METHOD}

Consider an array of $N$ generally non-identical elements (such as for arrays comprising multiple element types as studied here). The array far-field function can then be written as

$$
\boldsymbol{f}(\hat{\boldsymbol{r}})=\sum_{n=1}^{N} w_{n} \boldsymbol{f}_{n}(\hat{\boldsymbol{r}}) \quad \text { with } \quad \boldsymbol{f}_{n}(\hat{\boldsymbol{r}})=\boldsymbol{f}_{n}^{0}(\hat{\boldsymbol{r}}) e^{j k \boldsymbol{r}_{n} \cdot \hat{\boldsymbol{r}}},
$$

where $w_{n}$ is the complex excitation coefficient of the $n$th element, $k$ is the wavenumber, $\left\{\boldsymbol{f}_{n}(\hat{\boldsymbol{r}})\right\}_{n=1}^{N}$ is the set of embedded far-field vector element patterns for the elements placed at the locations $\left\{\boldsymbol{r}_{n}\right\}_{n=1}^{N}$ and the direction $\hat{\boldsymbol{r}}(\theta, \phi)=$ $\sin (\theta) \cos (\phi) \hat{\boldsymbol{x}}+\sin (\theta) \sin (\phi) \hat{\boldsymbol{y}}+\cos (\theta) \hat{\boldsymbol{z}}$. Now, for convenience, let us also introduce the $N$-dimensional excitation vector $\mathbf{w}=\left[w_{1}, w_{2}, \ldots, w_{N}\right]^{T}$, where ${ }^{T}$ denotes the transpose.

\section{A. Aperiodic array synthesis by Compressive Sensing (CS)}

The proposed approach is based on the CS methodology, where the problem of designing a maximally sparse array is formulated as finding the element excitation vector with a minimum number of non-zero entries - i.e. $\operatorname{argmin}\|\mathbf{w}\|_{\ell_{0}}$ with $\mathbf{w} \in \mathbb{C}^{N}$ - while fulfilling certain pattern constraints. Although this is an NP-hard (Nondeterministic Polynomial time hard) problem, it can be solved efficiently for large $N$ by approximating the $\ell_{0}$-norm minimization through an iterative weighted $\ell_{1}$-norm minimization procedure [16]. Typically, this 
numerically efficient procedure requires only few iterations for the excitation vector to converge and provides good accuracy for complex beam shaping specifications [12] and also multibeam scenarios [13] as considered in this paper. For the latter scenarios, the $i^{\text {th }}$ iteration of the optimization algorithm can be mathematically written as [13]:

$$
\begin{aligned}
& \underset{\mathbf{w}^{i} \in \mathbb{C}^{N}}{\operatorname{argmin}}\left\|\mathbf{Z}^{i} \mathbf{w}^{i}\right\|_{\ell_{1}} \\
& \text { subject to } \begin{cases}f_{\mathrm{co}}^{p}\left(\hat{\boldsymbol{r}}_{\mathrm{p}}\right)=1, & p=1 \\
\left|f_{\nu}^{p}(\hat{\boldsymbol{r}})\right|^{2} \leq M_{\nu}^{p}(\hat{\boldsymbol{r}}), & p=1, \ldots, P\end{cases}
\end{aligned}
$$

where $\left\{M_{\nu}^{p}(\hat{\boldsymbol{r}})\right\}_{p=1}^{P}$ are the prescribed radiation masks for the the vector field component $\nu$ of the array far-field functions $f_{\nu}^{p}(\hat{\boldsymbol{r}})$ corresponding to $P$ beams scanning at the directions $\left\{\hat{\boldsymbol{r}}_{p}\right\}_{p=1}^{P}$, and the $m^{\text {th }}$ element of the diagonal matrix $\mathbf{Z}^{i}$ is given by $z_{m}^{i}=1 /\left(\left|w_{m}^{(i-1)}\right|+\epsilon\right)$. The matrix $\mathbf{Z}^{i}$ is chosen to effectively suppress redundant elements through magnifying their apparent contribution in the minimization process by an amount that is based on the previous solution $\mathbf{w}^{(i-1)}$. The parameter $\epsilon$ enables elements that are "turned off" to be engaged again later on during the iterative procedure, and is set to the value that is slightly smaller than the expected active element excitations.

\section{B. Multi-element array}

The shapes of the antenna element patterns strongly influence the optimum array layout and scanning performance, and therefore the elements should be chosen properly. In a simplified case of identical element patterns, Eq. (1) can be written as the product of the element pattern $f^{0}$ and the array factor $\mathrm{AF}$ :

$$
\boldsymbol{f}(\hat{\boldsymbol{r}})=\boldsymbol{f}^{0}(\hat{\boldsymbol{r}}) \mathrm{AF}(\hat{\boldsymbol{r}}) \quad \text { with } \quad \mathrm{AF}(\hat{\boldsymbol{r}})=\sum_{n=1}^{N} w_{n} e^{j k \boldsymbol{r}_{n} \cdot \hat{\boldsymbol{r}}}
$$

From the above expression one can see that the element pattern defines the envelope of the total array pattern and, hence, should ideally be uniform over the desired scanning range while having zero radiation outside. This would minimize the overall scan loss while suppressing the side lobe levels for the least number of elements. Some authors have investigated element types with such characteristics [17]. However, this choice can significantly compromise the gain in the boresight direction.

In practice, regardless of the element choice, a certain degree of gain loss at the maximum scan angle and radiation in unwanted directions will occur. A tradeoff between the side lobe suppression (minimum number of elements) and gain scan loss should therefore be considered when choosing the antenna element. We study array layouts employing multiple element types, characterized by different beamwidths, in order to simultaneously reduce the number of elements and gain scan losses with respect to commonly considered arrays with one element type only.

Extending the proposed CS approach to the synthesis of multi-element arrays is straightforward. Each element weight $w_{n}$ is associated with a given element type and position

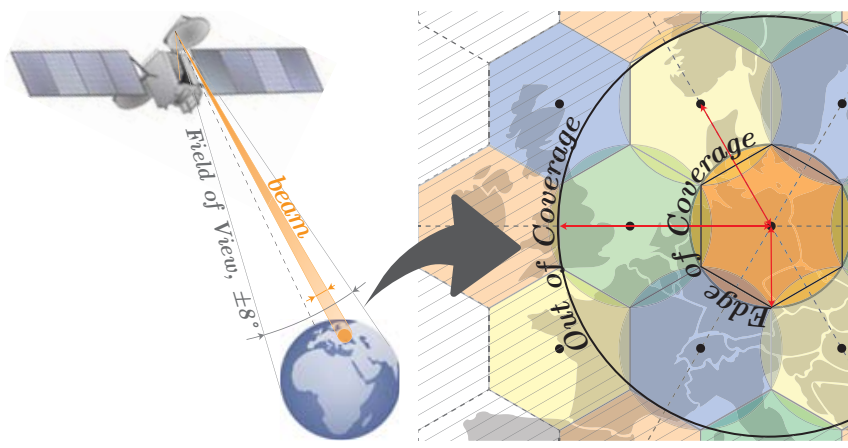

Fig. 1: Illustration of a multi-beam scenario for a SATCOM application. Each hexagon (insertion on the right) represents a (main-lobe) beam spot on the Earth and the colors distinguish the four frequency bands.

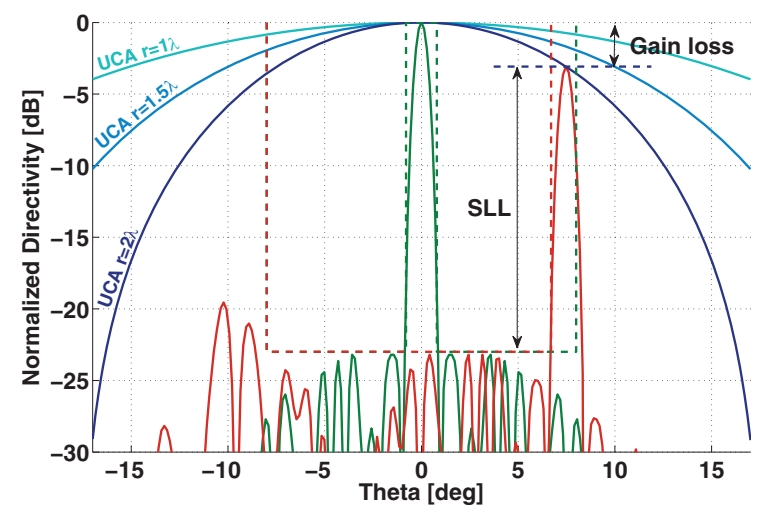

Fig. 2: Element pattern for UCAs of radii 1, 1.5 and $2 \lambda$ (from light to dark blue). Radiation pattern for broadside (green) and scanned beam (red) and respective radiation mask (dashed).

through the respective element pattern $\boldsymbol{f}_{n}(\hat{\boldsymbol{r}})$. To include multiple elements at the same time, one can sample the array aperture multiple times, once for every antenna type considered. It is also possible to only sample certain specific regions for a given element type. In this way, one can enforce the layout to consist of rings with increasingly larger elements, for instance, thus controlling the distribution of the elements on the aperture.

\section{RESULTS}

In GEO SATCOM applications multiple narrow spot-beams provide cellular-like coverage of the Earth surface, visible under a Field of View (FoV) of $\pm 8^{\circ}$ (Fig. 1). To satisfy the maximum allowed interference levels between the beams, multi-frequency arrangements of multiple beams (as indicated by different colors in Fig. 1) are used for beam to sidelobe isolation. For a 4-color hexagonal arrangement with an interbeam distance of $1.06^{\circ}$, the next iso-frequency beams appear at an Out of Coverage (OoC) angle of $0.795^{\circ}$ [13]. In the remaining part of the angular range, maximum radiation levels of $-20 \mathrm{~dB}$ are required to respect the allowed interference levels [17]. The radiated fields outside the FoV are directed toward the cold sky (low interference environment), and are 

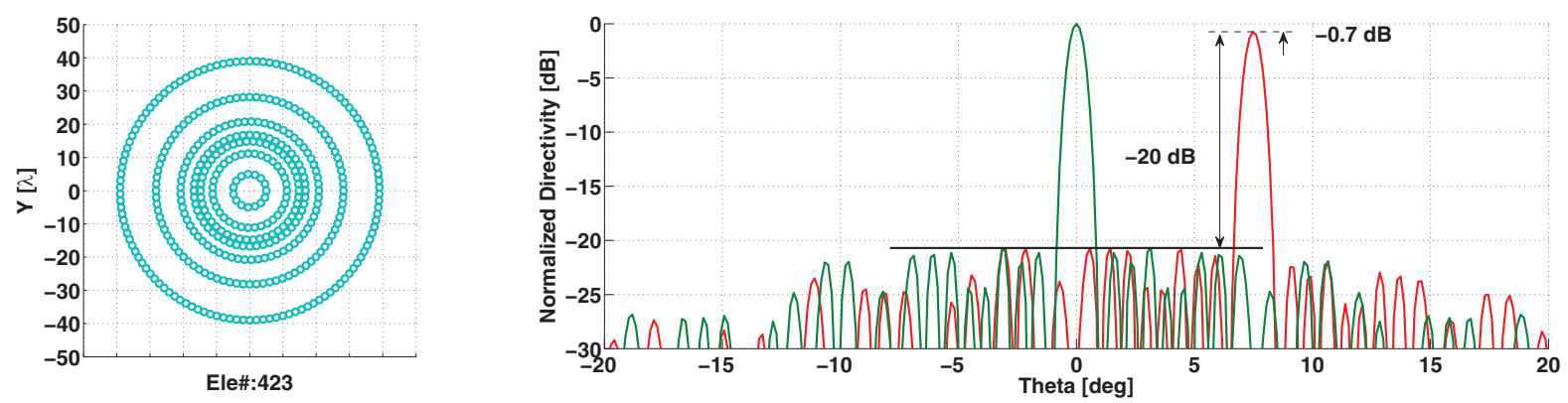

Fig. 3: Array layout and radiation pattern for UCA of radius $1 \lambda$
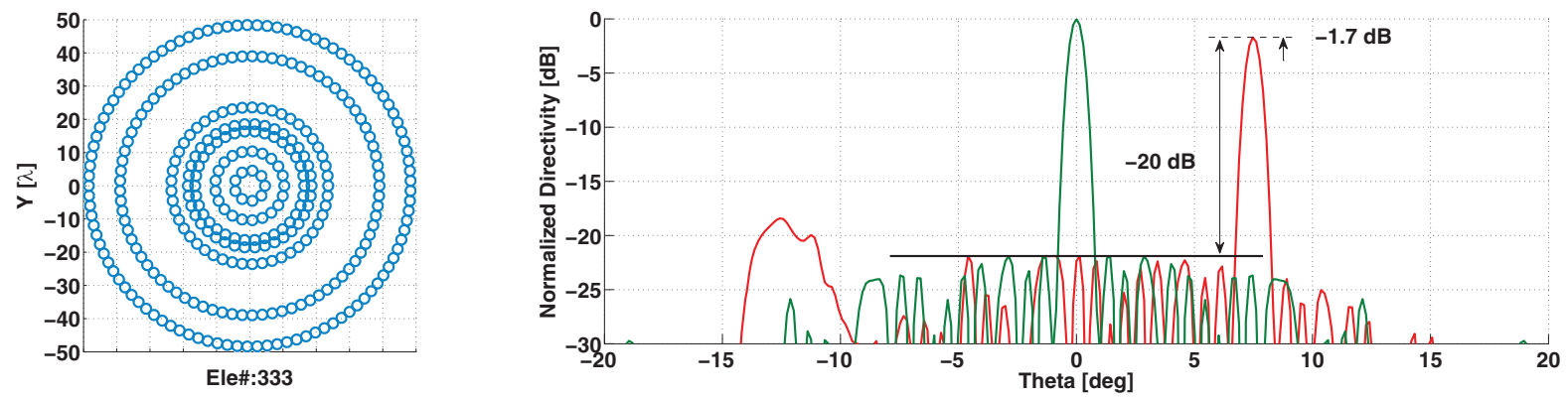

Fig. 4: Array layout and radiation pattern for UCA of radius $1.5 \lambda$
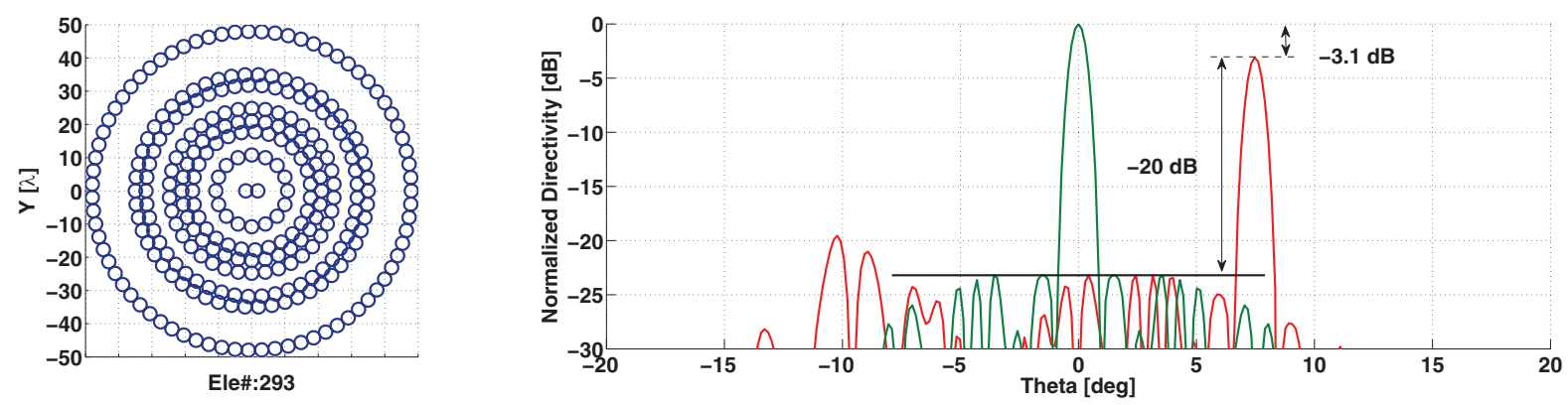

Fig. 5: Array layout and radiation pattern for UCA of radius $2 \lambda$
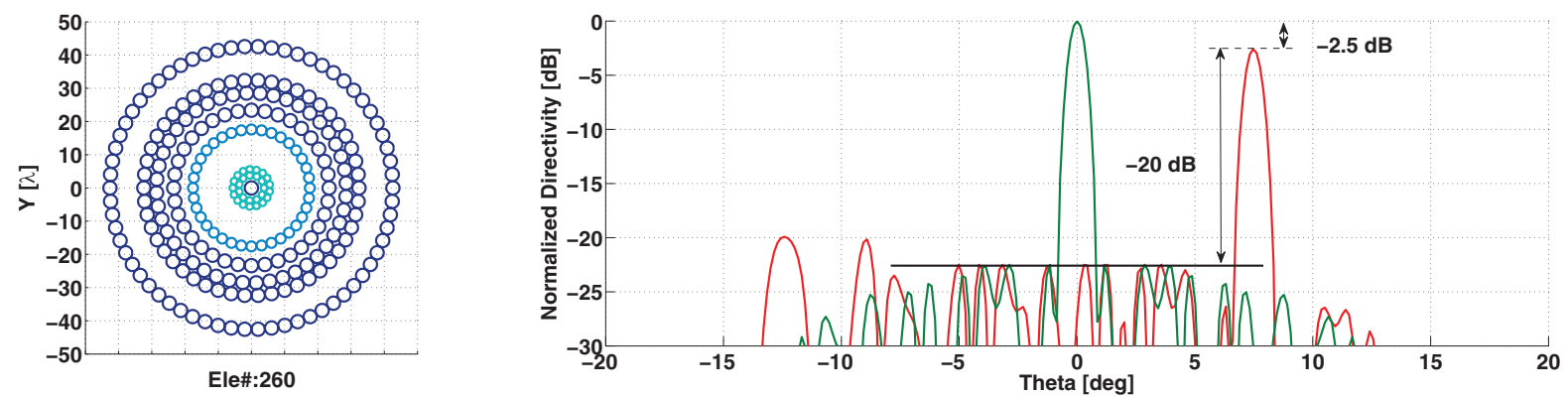

Fig. 6: Array layout and radiation pattern for multi element UCAs of radii $1,1.5$ and $2 \lambda$

therefore of minor concern. Accordingly, the required radiation mask to be defined for the optimization of a given beam should extend from its $\mathrm{OoC}$ angle up until the edge of the $\mathrm{FoV}$, as shown in Fig. 2.

The array has been designed for scanning capabilities over the entire FoV by optimizing both the broadside and the faroff beam $\left(\theta_{m}=7.5^{\circ}\right)$ [13]. The array has a total aperture of $100 \lambda$, which, if uniformly sampled with the inter-element distance of $3 \lambda$ (to avoid grating lobes in the FoV), would require 1245 elements arranged in 20 rings. The elements chosen for this study are Uniform Circular Apertures (UCAs) of different radii. Although the UCA represents a theoretical model, its radiation pattern behaves similar to that of typical horn antenna of the same dimension. Also, the UCA can be modeled with a simple analytical expression providing the values of the beam directivity and pattern shape for a given aperture area. According to the specifications for the desired scan range, three radii have been considered: $1,1.5$ and $2 \lambda$ 
with the corresponding directivities of about 16, 20 and 22 $\mathrm{dBi}$ and relative gain taper levels at $\theta_{m}=7.5^{\circ}$ of $0.7,1.1$ and $3.1 \mathrm{~dB}$, as illustrated in Fig. 2.

Three array layouts have been optimized for each of the three element types; their combination constitutes the fourth case. Figures 3, 4 and 5 show the layouts and corresponding E-plane far-field pattern cuts for the center and most scanned beams. As expected, the least number of elements is achieved for the largest UCA, 293 elements, so is maximum the gain loss, which is as large as $\sim 50 \%$. The array that has been designed using all the three element types is shown in Fig. 6. The resulting array further reduces both the number of elements to 260 (28 small, 36 medium, and 196 large element) and the scan loss to $-2.5 \mathrm{~dB}$. The improvement in array design and performance can also be observed by comparing the results in Fig. 6 to previous publications (e.g. [17]).

\section{CONCLUSIONS}

The herein presented CS-based array synthesis approach has been found to be a general and flexible optimization tool for large-scale sparse arrays employing multiple types of antenna elements. It is well-suited for applications involving complex pattern shapes and multi-beam specifications, where a high degree of modularity in the design is required. The possibility of minimizing the gain degradation loss when scanning and further reducing the number of elements (through the optimized combination of different element dimensions) was demonstrated for a circular aperture array with a diameter of $100 \lambda$. Modularity in this design has been achieved by enforcing the element arrangement in concentric rings. This approach can be easily extended to introduce additional degrees of freedom, such as to control the number of element excitation levels during the array synthesis procedure - which is what we plan to do in future studies.

\section{ACKNOWLEDGMENTS}

This work has been funded by Swedish Governmental Agency for Innovation Systems VINNOVA through the CHASE Antenna Systems Excellence research centre at Chalmers.

\section{REFERENCES}

[1] C. Bencivenni, M. Ivashina, and R. Maaskant, "A simple method for optimal antenna array thinning using a broadside maxgain beamformer," in Antennas and Propagation (EuCAP), 2013 7th European Conference on, April 2013, pp. 1799-1802.

[2] R. L. Haupt, "Thinned arrays using genetic algorithms," IEEE Trans. Antennas Propag., vol. 42, no. 7, pp. 993-999, 1994.

[3] M. M. Khodier and C. G. Christodoulou, "Linear array geometry synthesis with minimum sidelobe level and null control using particle swarm optimization," IEEE Trans. Antennas Propag., vol. 53, no. 8, pp. 2674-2679, 2005.

[4] Óscar Quevedo-Teruel and E. Rajo-Iglesias, "Ant colony optimization in thinned array synthesis with minimum sidelobe level," IEEE Antennas Wireless Propag. Lett., vol. 5, no. 1, pp. 349-352, 2006.

[5] G. G. Roy, S. Das, P. Chakraborty, and P. N. Suganthan, "Design of non-uniform circular antenna arrays using a modified invasive weed optimization algorithm," IEEE Trans. Antennas Propag., vol. 59, no. 1, pp. 110-118, 2011.

[6] Y. Liu, Z. Nie, and Q.-H. Liu, "Reducing the number of elements in a linear antenna array by the matrix pencil method," Antennas and Propagation, IEEE Transactions on, vol. 56, no. 9, pp. 2955-2962, 2008.
[7] G. Oliveri, M. Donelli, and A. Massa, "Linear array thinning exploiting almost difference sets," IEEE Trans. Antennas Propag., vol. 57, no. 12, pp. 3800-3812, 2009.

[8] D. Caratelli and M. Vigano, "A novel deterministic synthesis technique for constrained sparse array design problems," IEEE Trans. Antennas Propag., vol. 59, no. 11, pp. 4085-4093, Nov 2011.

[9] A. Ishimaru, "Unequally spaced arrays based on the poisson sum formula," IEEE Trans. Antennas Propag., vol. 62, no. 4, pp. 1549-1554, April 2014

[10] W. P. M. N. Keizer, "Large planar array thinning using iterative fft techniques," IEEE Trans. Antennas Propag., vol. 57, no. 10, pp. 33593362, Oct 2009.

[11] O. Bucci, S. Perna, and D. Pinchera, "Advances in the deterministic synthesis of uniform amplitude pencil beam concentric ring arrays," IEEE Trans. Antennas Propag., vol. 60, no. 7, pp. 3504-3509, 2012.

[12] S. E. Nai, W. Ser, Z.-L. Yu, and H. Chen, "Beampattern synthesis for linear and planar arrays with antenna selection by convex optimization," IEEE Trans. Antennas Propag., vol. 58, no. 12, pp. 3923-3930, 2010.

[13] C. Bencivenni, M. Ivashina, R. Maaskant, and J. Wettergren, "Fast synthesis of wide-scan-angle maximally sparse array antennas using compressive-sensing and full-wave em-analysis," IEEE Trans. Antennas Propag., Submitted 2015.

[14] — , "Design of maximally sparse antenna arrays in the presence of mutual coupling," IEEE Antennas Wireless Propag. Lett., vol. 14, pp. 159-162, 2015.

[15] P. Angeletti, G. Toso, and G. Ruggerini, "Sparse array antennas with optimized elements positions and dimensions," in Antennas and Propagation (EuCAP), 2014 8th European Conference on, April 2014, pp. 3142-3145.

[16] G. Prisco and M. D’Urso, “Maximally sparse arrays via sequential convex optimizations," IEEE Antennas Wireless Propag. Lett., vol. 11, pp. 192-195, 2012

[17] O. Bucci, T. Isernia, S. Perna, and D. Pinchera, "Isophoric sparse arrays ensuring global coverage in satellite communications," IEEE Trans. Antennas Propag., vol. 62, no. 4, pp. 1607-1618, April 2014. 